\title{
Supported Molybdenum Carbide Nanoparticles as Hot Hydrogen Reservoirs for Catalytic Applications
}

\author{
Marc Figueras ${ }^{\dagger}$, Ramón A. Gutiérrez, ${ }^{\ddagger}, \S$ Francesc Viñes, ${ }^{*}{ }^{\dagger}$ Pedro J. Ramírez, ${ }^{\S, £}$ Jose \\ Rodriguez, ${ }^{*}, \star$ Francesc Illas ${ }^{\dagger}$
}

\begin{abstract}
${ }^{\dagger}$ Departament de Ciència de Materials i Química Física \& Institut de Química Teòrica i Computacional (IQTCUB), Universitat de Barcelona. c/ Martí i Franquès 1-11, 08028 Barcelona, Spain.

$\$$ Chemistry Department, Brookhaven National Laboratory, Upton, NY 11973, United States.

$\S$ Facultad de Ciencias, Universidad Central de Venezuela, Caracas 1020-A, Venezuela.

${ }^{£}$ Present address: Zoneca-CENEX, R\&D Laboratories, Alta Vista, 64770 Monterrey, Mexico.
\end{abstract}

*Corresponding authors: José A. Rodriguez (rodriguez@bnl.gov), Francesc Viñes

(francesc.vines@ub.edu)

\section{Experimental Details}

For the preparation of the $\mathrm{MoC}_{\mathrm{y}} / \mathrm{Au}(111)$ systems, we followed a procedure described in previous studies. ${ }^{1,2}$ The $\mathrm{MoC}_{\mathrm{y}}$ nanoparticles were generated by deposition of Mo metal onto a reactive multilayer of ethylene, which was physisorbed on a $\mathrm{Au}(111)$ surface at $100 \mathrm{~K}$. Upon heating to $750 \mathrm{~K}$, part of the ethylene desorbed and the $\mathrm{MoC}_{\mathrm{y}}$ nanoparticles were left on the gold substrate. These transformations could be followed by using a combination of X-ray Photoemission Spectroscopy (XPS), Temperature Programmed Desorption (TPD), and Scanning Tunnelling Microscopy (STM).1.2 In this synthetic approach, it is possible to control the C/Mo ratio in the carbide nanoparticles, and this was varied between 0.6 and 1.1. No signal was observed in the O 1s XPS region indicating that the prepared NPs did not contain oxygen. ${ }^{3}$ Images acquired by STM showed that the generated nanoparticles were small (0.8-1.5 nm) and grew over the face-centred cubic $(f c c)$ troughs located on either side of the elbows of the reconstructed $\mathrm{Au}(111)$ substrate. ${ }^{1}$

The reactivity of the $\mathrm{MoC}_{\mathrm{y}} / \mathrm{Au}(111)$ surfaces towards $\mathrm{H}_{2}$ was examined in a system which combines an Ultra-High Vacuum ( $U H V$ ) chamber (base pressure $\sim 7 \cdot 10^{-10}$ mbar) and a batch reactor. 2 Within this system, the sample could be transferred between the reactor and UHV chamber without exposure to air. The UHV chamber was equipped with instrumentation for XPS, Ultraviolet Photoelectron Spectroscopy (UPS), LowEnergy Electron Diffraction (LEED), Ion Scattering Spectroscopy (ISS), and TPD. In 
the tests to study the interactions with hydrogen, $\mathrm{MoC}_{\mathrm{y}} / \mathrm{Au}(111)$ surfaces were positioned in the batch reactor at $\sim 300 \mathrm{~K}$, then 1 Torr of $\mathrm{H}_{2}$ was introduced for a period of five minutes. After this exposure, the hydrogen gas was removed from the reactor and, through differentially pumped stages, each sample was transferred back to the UHV chamber for surface characterization with XPS and TPD. The samples were free of oxygen and only contained $\mathrm{Mo}, \mathrm{C}$, and $\mathrm{H}$ on top of the gold substrate.

\section{Computational Details}

The hydrogen reservoir capabilities on $\mathrm{MoC}_{\mathrm{y}} / \mathrm{Au}(111)$ has been assessed using a stoichiometric $\mathrm{Mo}_{12} \mathrm{C}_{12}$ cluster supported on a $\mathrm{Au}(111)$ surface slab model. Such supported nanoparticle structures was obtained and characterized in a previous work,3 obtained first by a thorough search of cluster minima in vacuum, using shape templates from similar TiC compound gained by data mining searches combining interatomic potentials and Density Functional Theory (DFT) calculations, ${ }^{4}$ plus exhaustively sampling the attachment of such clusters on a $\operatorname{Au}(111)$ slab model of $p(6 \times 6)$ dimensions. The $\mathrm{Au}(111)$ slab model is four-layered, where the two upmost layers have been allowed to relax, while the bottommost two layers were kept frozen mimicking the bulk Au environment, i.e. 2+2 approach, built and relaxed from a previously optimized Au bulk using the very same computational scheme. Such large dimensions were found to be necessary to properly isolate $\mathrm{MoC}_{\mathrm{y}}$ clusters from periodically replicated images, and for the same purpose, a very large vacuum region of $30 \AA$ was added.

The DFT calculations were carried out using the Perdew-Burke-Ernzerhof $(P B E)^{5}$ exchange-correlation $(x c)$ functional, adding a dispersive forces treatment by Grimme D3 correction, ${ }^{6}$ already found to be accuracy-wise suited for such systems. 3 Spin-polarization was found to be negligible such past studies, 3 and so presently not accounted for. The effect of core electrons on the valence electron density has been described by the Projector Augmented Wave $(P A W)$ method of Blöchl, ${ }^{7}$ as implemented in the employed Vienna Ab-initio Simulation Package (VASP) code ${ }^{8}$ as implemented by Kresse and Joubert. ${ }^{9}$ Optimizations were carried out fully relaxing the aforementioned two gold layers, the attached $\mathrm{MoC}$ clusters, and the $\mathrm{H}_{x}(x=1,2)$ species upon. The electronic and atomic optimization criteria were set to $10^{-5} \mathrm{eV}$ and $0.01 \mathrm{eV} \cdot \AA^{-1}$, respectively, that together with a basis set of plane waves with a kinetic energy limit of $415 \mathrm{eV}$ and a $3 \times 3 \times 1 \mathbf{k}$-point mesh ensure results well within the chemical accuracy of 1 $\mathrm{kcal} \cdot \mathrm{mol}^{-1}$, i.e. below $\sim 0.04 \mathrm{eV}$. Transition State $(T S)$ structures were acquired by means 
of the Climbing-Image Nudged-Elastic-Band (CI-NEB) method, ${ }^{10}$ and adsorption minima and TSs were characterized as so by a frequency analysis carried out by Hessian matrix building and diagonalization, constructed by finite differences of $0.03 \AA$ in length.

Several initial geometries were tested for the $\mathrm{MoC}_{\mathrm{y}}$ nanoparticle, and the most stable structure was chosen to study the $\mathrm{H}_{x}$ adsorption, the $\mathrm{H}_{2}$ dissociation, and the $\mathrm{H}$ diffusion. The optimization procedure used to gain the minimum energy structure of the nanoparticles was also used to optimize the geometry of the different species adsorbed on the supported nanoparticles. Note that, due to the large number of possible topologically distinct sites a considerable number of geometry optimization runs was necessary. 
Figure S1. Amounts of $\mathrm{CH}_{\mathrm{x}}$ species generated on $\mathrm{MoC}_{1.1} / \mathrm{Au}(111)$ and $\mathrm{MoC}_{0.6} / \mathrm{Au}(111)$ after several cycles of hydrogen adsorption ( 1 Torr of $\mathrm{H}_{2}, 300 \mathrm{~K}, 5$ minutes) and desorption (heating at $500 \mathrm{~K}$ ).

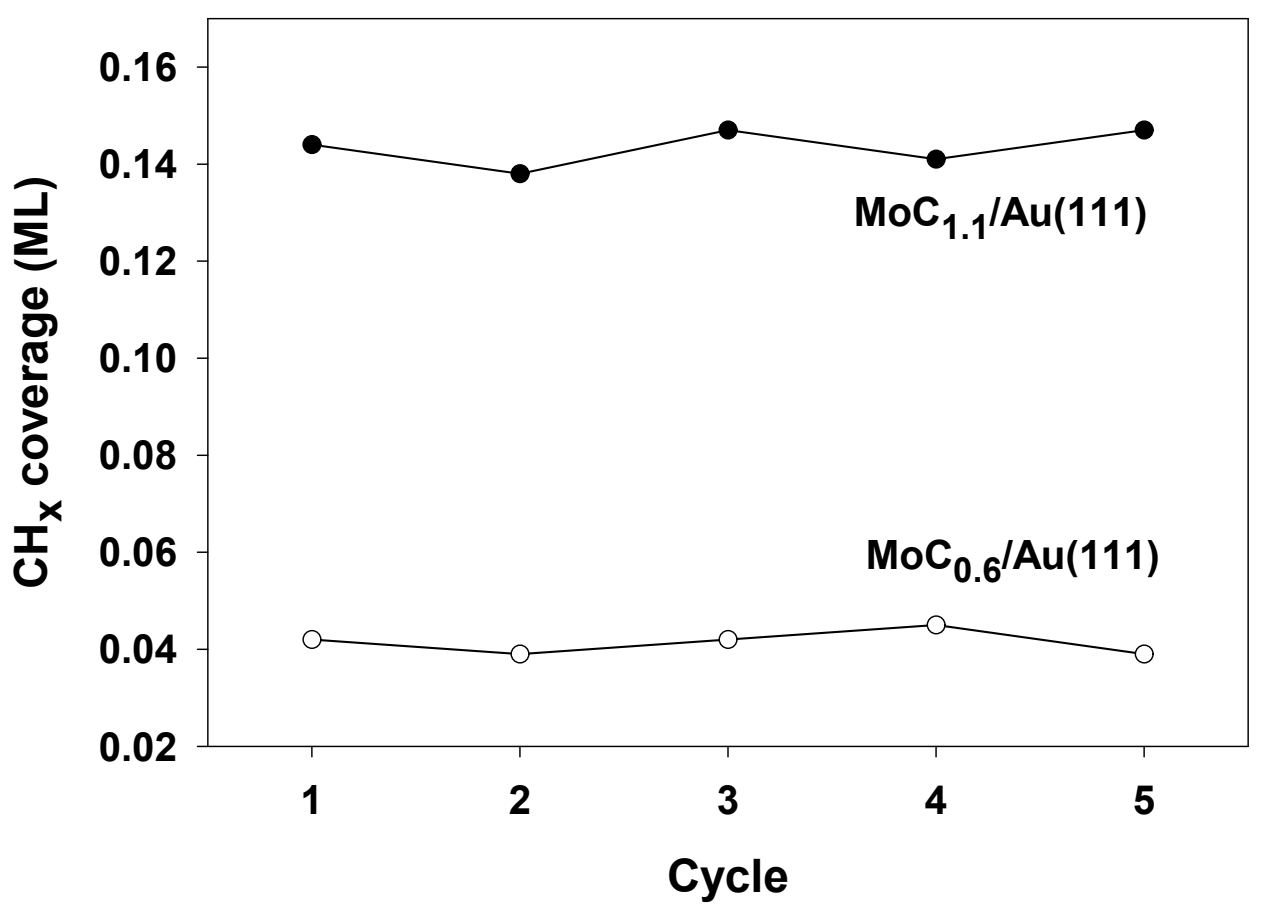




\section{References}

(1) Horn, J. M.; Song, Z.; Potapenko, D. V.; Hrbek, J.; White, M. G. Characterization of Molybdenum Carbide Nanoparticles Formed on Au(111) Using Reactive-Layer Assisted Deposition. J. Phys. Chem. B 2005, 109, 44-47.

(2) Posada-Pérez, S.; Ramírez, P. J.; Evans, J.; Viñes, F.; Liu, P.; Illas, F.; Rodriguez, J. A. Highly Active $\mathrm{Au} / \delta-\mathrm{MoC}$ and $\mathrm{Cu} / \delta$-MoC Catalysts for the Conversion of $\mathrm{CO}_{2}$ : The Metal/C Ratio as a Key Factor Defining Activity, Selectivity, and Stability. J. Am. Chem. Soc. 2016, 138, 8269-8278.

(3) Figueras, M.; Gutiérrez, R. A.; Prats, H.; Viñes, F.; Ramírez, P. J.; Illas, F.; Rodriguez, J. A. Boosting the Activity of Transition Metal Carbides Towards Methane Activation by Nanostructuring. Phys. Chem. Chem. Phys. 2020, 22, 7110-7118.

(4) Lamiel, O.; Bromley, S. T.; Illas, F. Low-energy Nanoscale Clusters of (TiC) $\mathrm{n} n=6$, 12: A Structural and Energetic Comparison with MgO. Theor. Chem. Acc. 2013, 132 , 1312.

(5) Perdew, J. P.; Burke, K.; Ernzerhof, M. Generalized Gradient Approximation Made Simple. Phys. Rev. Lett. 1996, 77, 3865-3868.

(6) Grimme, S.; Antony, J.; Ehrlich, S.; Krieg, S. A. A Consistent and Accurate $A b$ Initio Parametrization of Density Functional Dispersion Correction (DFT-D) for the 94 Elements H-Pu. J. Chem. Phys. 2010, 132, 154104.

(7) Blöchl, P. E. Projector Augmented-Wave Method. Phys. Rev. B 1994, 50, 17953 17979.

(8) Kresse, G.; Furthmüller, J. Phys. Rev. B: Condens. Matter Mater. Phys. 1996, 54, 11169-11186.

(9) Kresse, G.; Joubert, D. From Ultrasoft Pseudopotentials to the Projector Augmented-Wave Method. Phys. Rev. B 1999, 59, 1758-1775.

(10) Henkelman, G.; Uberuaga, B. P.; Jónsson, H. A. A Climbing Image Nudged Elastic Band Method for Finding Saddle Points and Minimum Energy Paths. Phys. Rev. B:

Condens. Matter Mater. Phys. 2000, 113, 9901-9904. 